\title{
A Cooperative High-Speed Positioning Control System for Dual-Axis Permanent-Magnet Linear Motor
}

\author{
Chao Zhang(1), Yudan Yang(2), Su Pan(3) \\ (1) Electrical and Electronic Engineering Department, Changchun University, Changchun, CHINA \\ (2) Scientific Research Center, China-Japan Union Hospital of Jilin University, Changchun, CHINA \\ (3) Department of Orthopedics, Second Hospital of Jilin University, Changchun, CHINA
}

\section{SUMMARY}

The Smart Cross Platform guarantees content compatibility of various smart devices and supports numerous programming languages, which is a solution to increasing development productivity. The SVM (Smart Virtual Machine) is the core virtual machine of the smart cross platform. This paper presents a technology which allows the SVM previouslyoperating on smart devices to operate in the web environment. In the whole design and implementation of the SVM operating in the web based environment, this paper focuses on the interpreter, which is used as a method to execute the contents.

KEY WORDS: smart cross platform; SVM (smart virtual machine); interpreter, servosystem; linear motor.

\section{INTRODUCTION}

The quantum leaps in power electronics, machining, sensor and digital technologies have made the high-speed, high-precision, intelligent, environmentally-friendly and multi-functional compound machine tools become the mainstream among the CNC machine tools; however, the traditional drive technology can no longer meet the performance requirements for high speed and high precision, especially for the multi-functional compound machining centre [1], as it involves many intermediate components and large movement inertia and thus brings about some nonlinear effects, such as elastic deformation, reverse gap, friction, shock, response delay and stiffness reduction. To solve the problem, the direct drive mode represented by the linear motor drive has gained significant attention from scholars and manufacturers.

Thanks to its advantages like high efficiency and precision over the traditional servosystems, the linear motor has been widely applied in high-precision motion control systems. However, the motor will still be affected by various interferences during operation, including external 
factors, such as current ripples, and internal ones, such as magnetic thrust fluctuation, cogging force fluctuation and end effect. In the high-precision servosystem of a dual-axis permanentmagnetic linear motor, it is necessary to precisely identify and eliminate these interferences and obtain better control in order to achieve cooperative high-speed positioning. In high-precision servosystems, such as robots and aerospace systems, the sliding mode variable structure control algorithm is very robust against these various interferences. This paper puts forward a highprecision linear motor positioning control strategy based on the complex dynamic friction model. In this strategy, the system friction and positioning are treated as external interferences [2], and then the sliding mode variable structure control algorithm is adopted to estimate the uncertain parameters in the system [3].

Afterwards, the sliding mode variable structure algorithm is used to design the positioning controller. To effectively eliminate the buffeting in the sliding mode control, a continuous function is adopted to replace the switch function [4].

\section{MATHEMATICAL MODEL FOR PERMANENT-MAGNET SYNCHRONOUS LINEAR MOTOR}

The permanent-magnet synchronous linear motor is a complex nonlinear system, whose stator inductance, flux linkage and rotor position are complexly coupled. In addition, considering the impacts from saturation of magnetic circuit and mutual inductance between windings, it is difficult to establish a mathematical model for the permanent-magnet synchronous linear motor [5]. To help analyze the performance of linear motor, the following assumptions are made:

(1) The impact from the saturation of magnetic circuit in the motor is neglected; besides, the magnetic hysteresis and eddy-current loss of the stator core and the rotor core inside the motor are not taken into consideration.

(2) The three-phase motor windings are ideally and symmetrically distributed and the angle between each two adjacent axes is $120^{\circ}$.

(3) The changes in the stator electromotive force inside the motor follow the sine wave; besides, the impact from high-order harmonic waves inside the motor magnetic field is not taken into consideration.

\subsection{MATHEMATICAL MODEL UNDER THE DQ COORDINATE SYSTEM}

The mathematical model for linear motor under the $d q$ coordinate system:

The stator voltage equations:

$$
\left\{\begin{array}{l}
u_{d}=R_{s} i_{d}+p \phi_{d}-w_{e} \phi_{q} \\
u_{q}=R_{s} i_{q}+p \phi_{q}-w_{e} \phi_{d}
\end{array}\right.
$$

The stator magnetic flux equations:

$$
\left\{\begin{array}{l}
\phi_{d}=L_{d} i_{d}+\phi_{f} \\
\phi_{q}=L_{q} i_{q}
\end{array}\right.
$$

In the above equations, $u_{d}$ and $u_{q}$, and $\phi_{d}$ and $\phi_{q}$ refer to the stator voltage and magnetic flux vectors on the $d$ axis and $q$ axis, respectively; $n_{p}$ refers to the number of pole-pairs; $w_{e}$ refers to the synchronous angular velocity of the magnetic field; $s$ refers to the rotor displacement of the 
linear motor; and $p$ refers to the differential operator $d / d t$. It can be learnt from Eq. (2) that the magnetic flux and the current are effectively decoupled under the $d q$ coordinate system to help achieve the control on the motor.

The electromagnetic thrust of the linear motor is expressed as:

$$
\begin{aligned}
F_{e} & =P_{e} / v_{m}=\frac{3}{2} n_{p} \frac{\pi}{\tau}\left(\phi_{d} i_{q}-\phi_{q} i_{d}\right) \\
& =\frac{3}{2} n_{p} \frac{\pi}{\tau}\left[\phi_{f} i_{q}+\left(L_{d}-L_{q}\right) i_{d} i_{q}\right]
\end{aligned}
$$

The electromagnetic thrust can be expressed as:

$$
F_{e}=P_{e} / v_{m}=\frac{3}{2} n_{p} \frac{\pi}{\tau} \phi_{f} i_{q}=k_{F} i_{q}
$$

where, $k_{F}$ refers to the electromagnetic thrust coefficient. At this time, the electromagnetic thrust $F_{e}$ is positively proportional to the $q$-axial current $i_{q}$. Therefore, the electromagnetic thrust $F_{e}$ can be controlled by adjusting the magnitude of $i_{q}$.

Mechanical motion equation reads:

$$
F_{e}=M_{n} p v_{m}+B_{n} v_{m}+F_{f}+F_{r}+F_{d}
$$

where $M_{n}$ refers to the equivalent mass, $B_{n}$ the friction coefficient, and $F_{f}, F_{r}$ and $F_{d}$ the nonlinear friction force, fluctuation force and external loading interference force, respectively.

\subsection{MATHEMATICAL MODEL UNDER AB COORDINATE SYSTEM}

The direct thrust control and speed sensorless estimation of the linear motor are achieved when the stator magnetic flux is under the $\alpha \beta$ coordinate system. The dynamic mathematical model is as follows:

The stator voltage equations:

$$
\left\{\begin{array}{l}
u_{\alpha}=R_{s} i_{\alpha}+p \phi_{\alpha} \\
u_{\beta}=R_{s} i_{\beta}+p \phi_{\beta}
\end{array}\right.
$$

The stator flux linkage equations:

$$
\left\{\begin{array}{l}
\phi_{\alpha}=L_{s} i_{\alpha}+\phi_{f} \cos \theta_{e} \\
\phi_{\beta}=L_{s} i_{\beta}+\phi_{f} \cos \theta_{e}
\end{array}\right.
$$

The voltage equations are obtained as follows:

$$
\left\{\begin{array}{c}
u_{\alpha}=R_{s} i_{\alpha}+L_{s} p i_{\alpha}-\frac{\pi}{\tau} n_{p} \phi_{f} v_{m} \sin \theta_{e} \\
u_{\beta}=R_{s} i_{\beta}+L_{s} p i_{\alpha \beta}+\frac{\pi}{\tau} n_{p} \phi_{f} v_{m} \cos \theta_{e}
\end{array}\right.
$$

The electromagnetic thrust is expressed as follows:

$$
F_{e}=\frac{3}{2} \frac{\pi}{\tau} n_{p}\left(\phi_{\alpha} i_{\beta}-\phi_{\beta} i_{\alpha}\right)
$$

where $u_{\alpha}, u_{\beta}, i_{\alpha}, i_{\beta}, \phi_{\alpha}$, and $\phi_{\beta}$ are respectively the electronic voltage, current and flux linkage of the $\alpha$ and $\beta$ axes, and the rest of the parameters are the same as above. 


\subsection{INTERFERENCE FACTORS TO THE PERMANENT-MAGNET SYNCHRONOUS LINEAR MOTOR SERVOSYSTEM AND THEIR COMPENSATION METHODS}

When the linear motor is located in a high-precision positioning system, effective compensation measures should be taken to handle various interferences existing in the linear motor to improve the dynamic performance of the servosystem.

\section{(1) Loading interference}

Load changes will cause the motor speed to fluctuate, thus decreasing the system performance. The commonly-used suppression method against loading interference is to adopt a state observer or the parameter identification method to observe and identify these interferences and then carry out real-time compensation.

\section{(2) Friction interference}

Friction is the interference entering the system from outer environment. In a linear motor servosystem, friction has the most obvious influence on its low-speed performance. The friction model can be simplified or improved according to the control requirements, and friction compensation is adopted to improve the motion performance of the control system.

(3) Positioning interference

Positioning interference is generated by the mutual interaction between the rotor core current and the stator magnetic field. On the basis of theoretical analysis and experimental research, the positioning interference is simplified as a sine wave with a certain period. The main compensation method for positioning interference is to firstly identify the interference and then compensate for it, or to treat it as an external interference and then effectively compensate for it using the modern control theories.

\section{DEFINITION AND MODEL OF THE CONTOUR ERROR OF DUAL-AXIS WORKBENCH}

The dual-axis workbench for the linear motor can achieve planar movement. The definition and model of its contour error are established in the bi-dimensional plane of the $X-Y$ coordinate system.

\subsection{DEFINITION OF CONTOUR ERROR}

The contour error of the dual-axis workbench for the linear motor originates from the tracking error of the single-axis servosystem. Under this premise, the actual positioning point deviates from the expected contour curve and generates contour errors because the dynamics of these two axes do not match.

The curve is the expected contour trajectory of the workbench during the operation process. At the time $t$, the expected point for the tool is denoted as point $R$. Considering that tracking errors exist in the single-axis servosystem, the actual point where the tool is located is set as $P(x, y)$. The line segment $P R$ is the tracking error of the workbench, $P Q$ the tracking error of the $X$-axial servosystem $E x$ and $R Q$ the tracking error of the $Y$-axial servosystem $E y$. The line segment $P N$ refers to the distance from point $P$ to the expected contour curve, and also the contour error $\varepsilon$. In order to achieve the contour error, the magnitude of error generated by the workbench during the operation process must be obtained first and only then it can be controlled in order 
to decrease the contour error. Considering that the contour error originates from the tracking error of the single-axis servosystem, the expression of the contour error can be obtained according to their geometrical relation so as to facilitate the real-time calculation of the contour size.

\subsection{CONTOUR ERROR}

(1) Linear contour error

During the operation of the dual-axis workbench for the linear motor, linear cutting is the most common and simplest operation state. In this state, the contour error model can be read more easily. The line segment $O R$ refers to the input linear trajectory of the numerically-controlled system. Let the angle between the line segment $O R$ and the $X$ axis be $\theta$. Suppose that point $R$ is the expected point for the tool at time $t$, and that Point $P$ is the actual point where the tool is located. Then, the distance $P R$ between point $P$ and point $R$ at this time refers to the system tracking error while the distance $P N$ between point $P$ and the expected line $O R$, the contour error $\varepsilon$.

The linear trajectory contour error equation can be obtained from the geometrical relation shown in the figure. The steps are as follows. Firstly, draw the following auxiliary lines - a line from $Q$ which is perpendicular to $O R$ and intersects with $O R$ at point $M$; and one from $P$ which is perpendicular to $Q M$ and intersects with $Q M$ at point $S$. Then, according to the geometric relation, we have:

$$
\begin{aligned}
& \varepsilon=P N=M S \\
& M S=Q M-Q S \\
& Q M=Q R \cdot \cos \theta=E_{y} \cos \theta \\
& Q S=P Q \cdot \sin \theta=E_{y} \sin \theta
\end{aligned}
$$

From the above Eq. (10), the contour error equation can be deduced:

$$
\varepsilon=E_{y} \cos \theta-E_{y} \sin \theta
$$

(2) Contour error of a random curve

The circular contour error model is taken in this paper for the contour error of a random curve. Suppose that the tool is expected to be located at point $R$ on the curve at the time $t$. Draw an inscribed curvature circle from point $R$ with its curvature radius. The curvature radius is denoted as $r$ and the circle centre as $(X O, Y O)$. At this time, the tool is actually located at point $P$, the line segment $P R$ is the system tracking error and the distance from point $P$ to the curve $\varepsilon$, the contour error.

According to the expression of the circular contour error Eq. (11), the contour error of a random curve is:

$$
\varepsilon \approx e_{r}=\sqrt{\left(x_{p}-x_{0}\right)^{2}+\left(y_{p}-y_{0}\right)^{2}}-r
$$

where, $x_{p}$ and $y_{p}$ are known quantities while $x_{0}$ and $y_{0}$ are unknown. Therefore, we need to obtain the expressions of $x_{0}$ and $y_{0}$. Draw a line tangent to the curve from point $R$, which has an angle of $\theta$ from the $X$ axis. From the geometrical relation, it can be learnt that:

$$
\left\{\begin{array}{l}
X_{0}=X_{R}-r \sin \theta \\
Y_{0}=Y_{R}+r \cos \theta
\end{array}\right.
$$


where, $r$ is the radius of curvature. According to the radius of curvature equation:

$$
r=\frac{1}{\rho}=\frac{\sqrt{\left(1+\left(\frac{d x}{d y}\right)^{2}\right)^{3}}}{\frac{d^{2} y}{d x^{2}}}
$$

From the above equation, $\varepsilon$ can be deduced as follows:

$$
\varepsilon \approx e_{r}=\sqrt{\left(X_{p}-X_{r}+r \sin \theta\right)^{2}+\left(Y_{p}-Y_{r}-r \cos \theta\right)^{2}}-r
$$

In this equation $X_{p}-X_{r}=E_{X}$, so:

$$
\varepsilon \approx e_{r} \sqrt{\left(\left(r \sin \theta+E_{x}\right)^{2}+\left(r \cos \theta-E_{y}\right)^{2}\right)}-r
$$

Then the Taylor expansion is:

$$
\varepsilon \approx e_{r}=\left(\sin \theta+\frac{E_{x}}{2 r}\right) E_{x}-\left(\cos \theta-\frac{E_{y}}{2 r}\right) E_{y}
$$

In the actual situation, $E_{x}$ and $E_{y}$ are very small compared to the radius of curvature $r$, and $\frac{E_{x}^{2}}{2 r}$ and $\frac{e_{y}^{2}}{2 r}$ are even closer to zero, so the expression can be simplified as:

$$
\varepsilon \approx E_{x} \sin \theta-E_{y} \cos \theta
$$

(3) Dual-axis independent contour control

Currently, the most widely applied method for dual-axis workbench contour control of a linear motor is to carry out independent control over each axis of the workbench. In this method, the controller is independently designed for each axis to improve the linear motor operation precision of each axis. This requires the linear motor servosystem to be highly precise, rapidly responsive and resistant to interferences [6].

According to the characteristics of the dual-axis workbench, the detailed parameters of each axis, the operation conditions of the linear motor and the actual work environment may vary. More importantly, the parameters of the workbench will change with the working conditions and the nonlinearity will change accordingly. So, it is very difficult to obtain the accurate parameters of the workbench and the system model. In addition, when the system is operating, there are all kinds of interferences, such as vibration, friction, load changes and so on, bringing different impacts on the operation of the two axes. However, the two axes are independently controlled, so it is difficult to further improve the contour accuracy.

In the system, when the single-axis tracking precision is improved, the system contour precision will not necessarily increase accordingly, because the contour precision of the dual-axis workbench is not equal to the tracking precision [7]. Moreover, as the input signals are different, the corresponding parameters of each axis will also vary. This phenomenon is particularly obvious in high-speed motions. The structure of the two separate axis contour control systems is shown in Figure 1. 


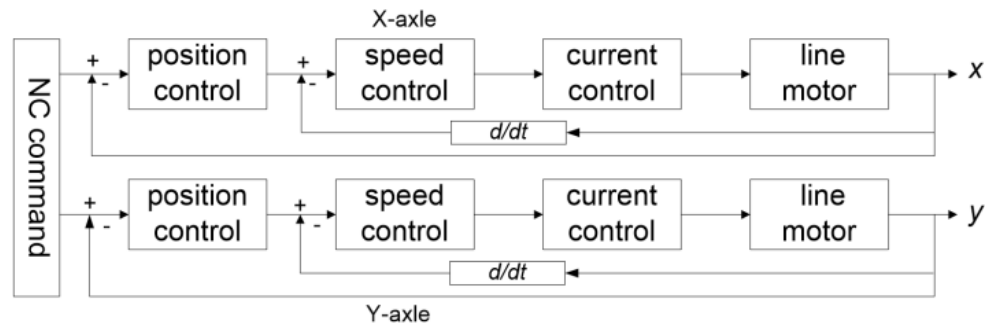

Fig. 1 Two independent control structure of $X-Y$ worktable

In Figure 1, the $X$-axis linear motor and the $Y$-axis linear motor each has a set of servo control system, which consists of position control, speed control and current control. However, the two servosystems are not connected. They move under the digital commands from the machine tool and subsequently output the displacements of the $X$-axis and the $Y$-axis, denoted as $x$ and $y$. The specific movement conditions of the two axes in the operation process are not considered.

After the contour curve is compensated for, the dual-axis linear motor control system sends the movement commands to the two axes, respectively. The movement of each axis is tracked by its control system, which focuses on the single-axis tracking precision. However, it is not considered whether the actual contour compounding the movements of the two axes complies with the expected contour.

\subsection{COOPERATIVE CONTOUR CONTROL}

The contour precision of the linear motor workbench servosystem is a main indicator of its performance. During the operation process, the contour error is compounded by the tracking error of each axis on the workbench [8]. There are many factors influencing the contour precision, such as vibration, load change, system delay and inappropriate dual-axis linear motor parameters, etc.

The previous workbench contour precision control methods mainly consider the single-axis situation. Under such methods, the whole workbench contour precision is controlled by improving the tracking precision of each single-axis linear motor with single-axis controllers. However, the contour precision can still be improved if the two axes can be controlled comprehensively. It has been demonstrated by practice that cooperative control is more effective in decreasing workbench contour errors than the single-axis independent control, except that it requires larger amount of calculation and a more complex controller structure. So in order to improve the workbench contour precision, cooperative contour control is adopted here to comprehensively control the two axes in addition to their independent controls. The core of cooperative contour control is to first comprehensively calculate the tracking errors of the $X$ axis and the $Y$-axis in the controller, then output the information obtained from the comprehensive calculation to the two axes respectively, and finally make additional compensation for the two workbench axes to improve the contour precision [9]. 
(1) Cooperative contour control system structure, including speed adjustment

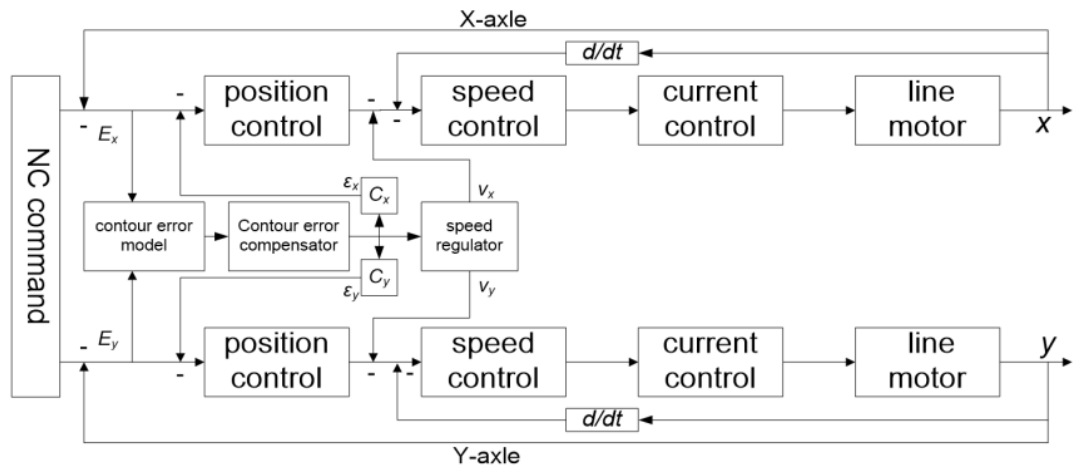

Fig. 2 Cross coupling structure of the contour control system including the speed regulator

As shown in Figure 2, the structure of the cooperative contour control system is different from the dual-axis independent contour control system.

(2) Selection of the contour control system structures

There are several contour control methods, whose advantages and disadvantages are compared and analyzed in Table 1. The first system, which has two independent axis controls, is of a simple structure and has no contour controller, but it aims at controlling the tracking errors, not contour errors. The cross-coupling control has a speed regulator to make the axial dynamic performance matching and at the same time to achieve contour error control, but the disadvantage is also obvious - the structure is too complex to be designed. The third structure has no speed regulator of cross coupling control. It can also achieve contour error control and is of a simple structure and easy to implement, but in theory the control effect is weaker than that of the speed regulation of cross coupling control.

Table 1 Comparison of the three contour control systems

\begin{tabular}{cccc}
\hline $\begin{array}{c}\text { Contour Control } \\
\text { System Structure }\end{array}$ & Advantage & Disadvantage & Performance \\
\hline $\begin{array}{c}\text { Two independent } \\
\text { control axes }\end{array}$ & $\begin{array}{c}\text { Simple structure and no contour } \\
\text { controller }\end{array}$ & $\begin{array}{c}\text { Aiming at controlling the } \\
\text { tracking error, not contour } \\
\text { error }\end{array}$ & $\begin{array}{c}\text { Low speed and } \\
\text { low precision }\end{array}$ \\
$\begin{array}{c}\text { Including a speed } \\
\text { regulator of cross } \\
\text { coupling control }\end{array}$ & $\begin{array}{c}\text { Contour error control taken into } \\
\text { account, and axial dynamic } \\
\text { performances matched through } \\
\text { speed regulation }\end{array}$ & $\begin{array}{c}\text { Too complex structure; } \\
\text { parameters of both axis servo } \\
\text { system to be considered; great } \\
\text { difficulty in design }\end{array}$ & $\begin{array}{c}\text { High speed and } \\
\text { high precision }\end{array}$ \\
$\begin{array}{c}\text { Including no speed } \\
\text { regulator of cross } \\
\text { coupling control }\end{array}$ & $\begin{array}{c}\text { Contour error control taken into } \\
\text { account, simple structure, } \\
\text { and ease of implementation }\end{array}$ & $\begin{array}{c}\text { In theory the control effect } \\
\text { weaker than that of the speed } \\
\text { regulation of cross coupling } \\
\text { control }\end{array}$ & High speed and \\
& high precision \\
\hline
\end{tabular}

In Figure 2, there is a cooperative contour controller between the servo control systems of the $X$-axis and the $Y$-axis. The $X$-axis and the $Y$-axis servosystems carry out position, speed and current control under digital commands and finally output the $x$-axis and $y$-axis displacements, denoted as $x$ and $y$. In the meantime, there is a cooperative contour controller between the servo control systems of the two axes. The input quantities to the controller are the tracking errors of the two axes, Ex and Ey. Through the calculation of the controller, the compensations of the two axes are respectively output to decrease the contour error. In the meantime, this controller 
contains a speed regulator, which is used to adjust the speeds of the two axes according to the contour error to match the dynamic response performances of the two axes and solve the contour error problem. It also contains a contour error model, a contour error compensator and contour error decomposers $C x$ and $C y$.

The causes for contour errors include dual-axis tracking errors and unmatchable dual-axis dynamic responses, so the contour control system in the figure reduces contour errors from these two aspects, i.e. outputting the position compensations to the two axes and then adjusting the dual-axis dynamic responses to match the use of the speed regulator.

Theoretically, this method can control the contour errors effectively, but it requires making contour error compensations as well as speed adjustments for both the $X$-axis and the $Y$-axis. Therefore, all parameters of the $X$-axis and $Y$-axis linear motor servosystems should be comprehensively considered during the design process. However, the dual-axis linear motor servosystem is a complex system, for which the comprehensive design can be very complicated and difficult, so scholars often neglect the speed adjustment part in the controller design.

Through comparison and analysis, this paper selects a common contour control system structure that can be relatively easily implemented. In the design of the contour control system, the $X$-axis and $Y$-axis linear motor servo control systems can be designed first and then the cooperative contour controller can be designed based on the above control systems. So in summary, the design process can be divided into two steps: firstly, design the single-axis controllers, then, design the cooperative contour controller.

(3) Design and planning of the cooperative contour controller

Figure 3 shows the basic internal structure of the cooperative contour controller, which consists of a contour error model, a contour error compensator and contour error decomposers $C X$ and $C y$.

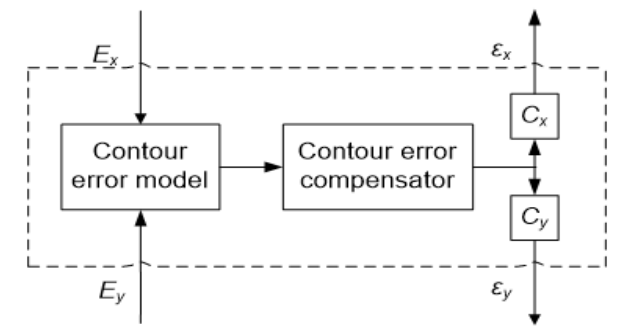

Fig. 3 Internal structure of the cross-coupling controller

The following should be considered during the design of the controller:

(1) Establishment of the contour error model;

(2) Design of the contour error compensator;

(3) Design of the contour error decomposers.

Contour error simulation during XY single-axis independent control

The mutual interactions between the single axes are neglected. In the dual-axis independent contour control method, it is assumed that the controller independently designed for each axis works under the following working conditions: circular contour movement at the radius of $0.15 \mathrm{~m}$ and the feed velocity of $2.2 \mathrm{rad} / \mathrm{s}$. This paper simulates the independent control over the dual-axis servosystem. 
As shown in the figure, when the system starts, the error is relatively large, which is quite similar to the actual situation. After the system becomes stable, the maximum contour error generated by the circular contour movement of the workbench is about $8 \mu \mathrm{m}$. This contour precision only reaches the average standard for a CNC machine tool and is far from the standard of $4.5 \mu \mathrm{m}$ required in this paper.

\section{HIGH-PRECISION OPTIMIZED POSITIONING CONTROL MODEL FOR DUAL- AXIS LINEAR MOTOR}

The motion and positioning precisions of the linear motor servosystem determine the machining precision, surface quality and production efficiency of a CNC machine tool, and the friction and positioning forces are the two key factors influencing the above two precisions. The positioning force severely affects the precise positioning of the system and might cause vibration and instability, especially under low-speed or light-load conditions. The strategy adopts a complex dynamic friction model to achieve cooperative high-speed positioning, which treats the system friction and positioning as external interferences and then uses the sliding mode variable structure control algorithm to estimate the uncertain parameters in the system. Afterwards, it uses the sliding mode variable structure algorithm to design the positioning controller. Regarding the buffeting in the sliding mode control, the switch function is replaced with a continuous function to effectively solve this problem.

The positioning force is often assumed as a periodical function changing with the position. Its mathematical expression is:

$$
F_{\text {cogging }}(y)=\sum_{i=1}^{\infty}\left(S_{i} \sin \left(\frac{2 i \pi}{p} y\right)+C_{i} \cos \left(\frac{2 i \pi}{p} y\right)\right)
$$

where, $p$ refers to the magnetic pole distance and $S$ and $C$ are constant coefficients. In actual practice, the most significant low-order terms in the expression are selected while other highorder ones are neglected. For example, $Z$ is selected from 1 to $n$ (finite value). However, the actual positioning force might not be totally periodical due to many complex physical influences, such as the differences between magnetic pole pairs. As a result, the periodical positioning force model is not able to describe the performance very precisely to certain extent. Some improvements are made to the above expression, including changing $S$ and $C$ from constants to position-dependent variables.

The $B$ spline function is applied to model the positioning force:

$$
\begin{gathered}
N_{j, k}(x)=\left\{\begin{array}{c}
1 \text { when } x \in\left(X_{j}, X_{j+1}\right), k=1 \\
0 \text { else }
\end{array}\right. \\
N_{j, k}(x)=\frac{x-X_{j}}{X_{j+k-1}-X_{j}} N_{j, k-1}(x)+\frac{X_{j+k-x}}{X_{j+k-} X_{j+1}} N_{j+1, k-1}(x), k \geq 2
\end{gathered}
$$

Considering that the increment in joint vector is the magnetic polar distance, the $B$ spline function value changes with each pair of poles, and thus the positioning force can be physically described as the change to sine wave amplitude of each pair of poles.

Considering that the $B$ spline function is positively proportional to the datum point coefficients $S$ and $C$, the mathematical model of periodical positioning force can be described in linear 
parameters. Therefore, the model can be greatly simplified to achieve the self-adaptive model compensation of positioning force.

\section{OVERALL STRUCTURE OF THE DUAL-AXIS LINEAR MOTOR POSITIONING CONTROL SYSTEM}

The permanent-magnet linear motor servo control system consists of a main signal processing circuit, a power source processing circuit, a main inverter circuit, an inverter drive circuit, a voltage and current sampling circuit as well as a protection circuit, as shown in Figure 4.

The core of the permanent-magnet linear motor servo positioning control system is the highspeed digital processor, as it helps obtain the rapid calculation of linear motor positioning control. The system control signal controls the primary winding of the linear motor through the inverter circuit and controls the movement track of the primary magnetic flux by changing the input three-phase voltage vector, thus acquiring control over the motor thrust.

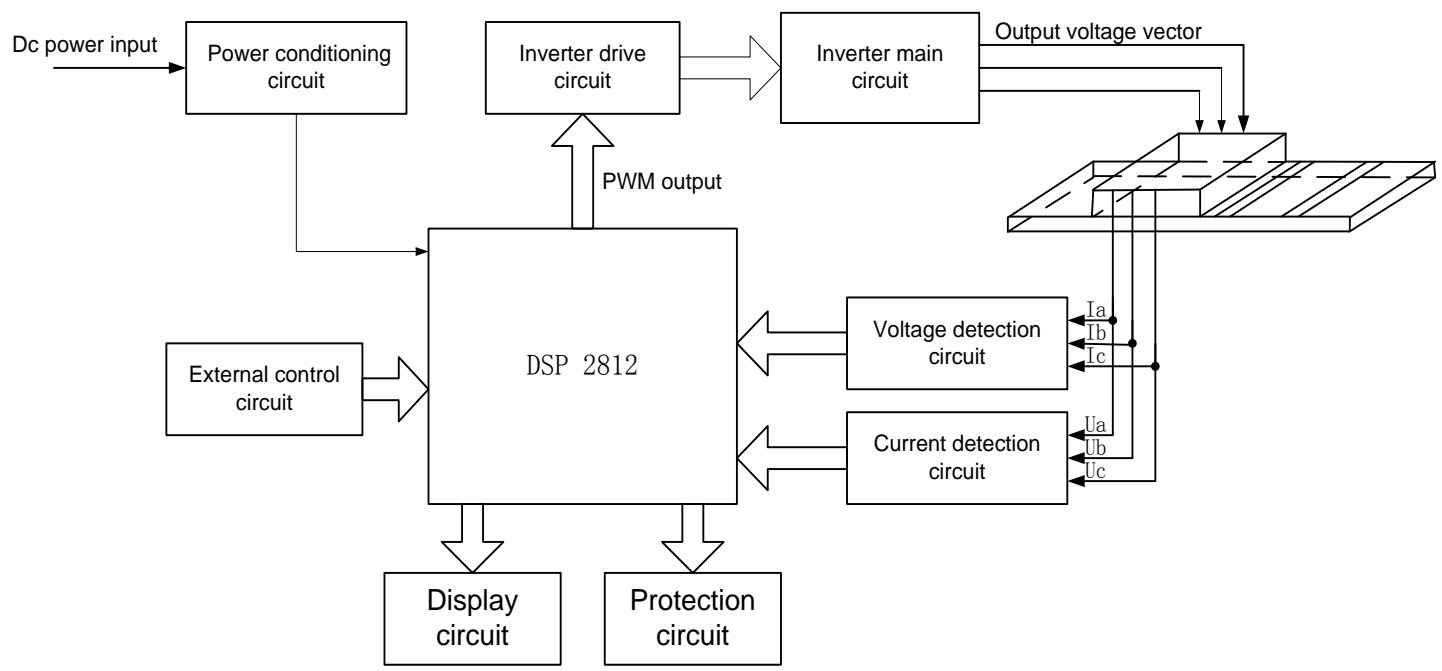

Fig. 4 Block diagram of the structure of the permanent-magnet linear synchronous motor servo control system

The principle for the direct thrust control technology is to start from the control effect and directly feed back the thrust to the controller for adjustment in the next period. The Hall sensor is used to detect the voltage and current of the primary winding so as to achieve the detection of signal quantity. In this way, the real-time feedback information about thrust is obtained based on the mathematical model of the permanent-magnet linear synchronous motor. Afterwards, the DSP event manager will output the PWM wave to control the inverter circuit and thus drive the linear motor. There are many different ways to control the speed of a DC motor, but one simple way is to use Pulse Width Modulation (PWM). The greater the pulse width is, the more average voltage will be applied to the motor terminals, and the stronger the magnetic flux will be inside the armature windings is the faster the motor will rotate, as shown below. The key to the whole closed-loop system is how to precisely detect the voltage and current of the primary winding. Another key point is to select the correct spatial voltage vector via the digital processor to control the inverter circuit. If these two problems are solved, the output thrust of the linear motor will strictly conform to the given quantity, and the servosystem will have high stability and a better tracking effect. 


\subsection{DESIGN OF SYSTEM INVERTER CIRCUIT}

The inverter is the core drive mechanism of the permanent-magnet linear motor servo control system. The digital signal processor can output the PWM signal to control the on/off switch of the MOS tube on each phase bridge in the inverter in order to control the voltage vector input into the primary winding. Considering that there is an integral relation between the voltage value and the magnetic flux of the primary winding and that the movement direction of the primary winding magnetic flux follows that of the voltage vector, the magnetic flux of primary winding can be controlled by adjusting the output voltage vector with the inverter. In this way, the control is achieved over the linear motor thrust. The principle is illustrated in Figure 5.

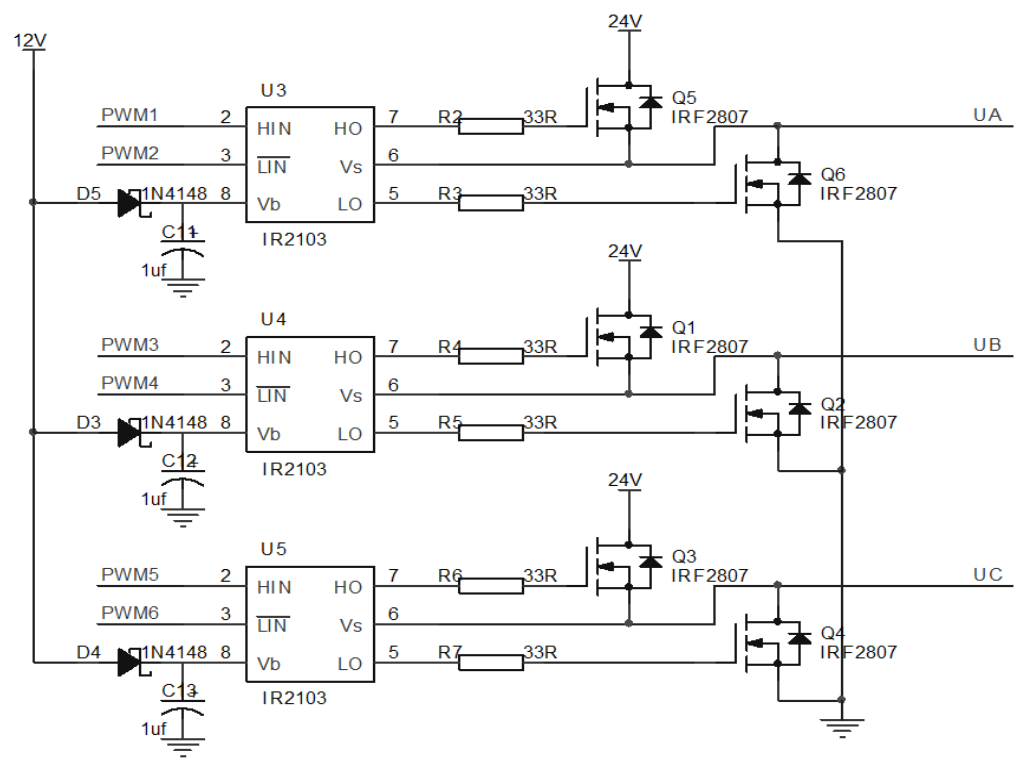

Fig. 5 Inverter circuit of the system

The inverter circuit adopts the three-phase bridge controllable circuit and drives the chip signal to make the MOS tube (quick switch type) switched on or off at different times, thus outputting the sine voltage wave. It should be noted that the dead time should be adjusted when the inverter circuit is being controlled. Each time when the upper and lower bridges take turns to switch on or off, there should be a certain time delay; or otherwise (the two MOS tubes on the same bridge switch on simultaneously) they will burn at the same time and affect the normal operation of the whole circuit. The three-phase output ports of the bridge circuit are directly connected to the primary winding of the linear motor.

\subsection{DESIGN OF THE DIRECT THRUST POSITIONING CONTROL}

After receiving control signals, this control system begins to execute direct thrust positioning control. Its control signal bit goes through the interruption detection. When there is a control command, the system firstly analyzes and calculates the current position and changes the amount of the primary magnetic flux and then calculates the actual state of the output thrust according to the current detection value of the primary winding, and acts in conjuction with the fuzzy control to give voltage vector control signal. Traditional control systems are built on mathematical models, in which the control system is described with one or more differential equations that define the system responses to its inputs. Such systems are often implemented 
as "PID controllers". The fuzzy control is the product of development and theoretical analysis for decades, and is highly effective. The software flowchart is shown in Figure 6.

Firstly, confirm if the system receives the control command. Upon confirmation that there is command, sample the current values and then determine the current magnetic flux state according to the relation between the current and the primary magnetic flux. In the meantime, calculate the current thrust. Then, calculate the three inputs of the fuzzy controller, including the current states of primary magnetic flux deviation, electromagnetic thrust deviation and primary magnetic flux angle, through the current feedback values. If any of these three inputs change, select the output voltage vector according to the fuzzy rule of the fuzzy controller and output it through PWM of the event manager. The output signal controls the MOS on the upper and lower bridges of the inverter.

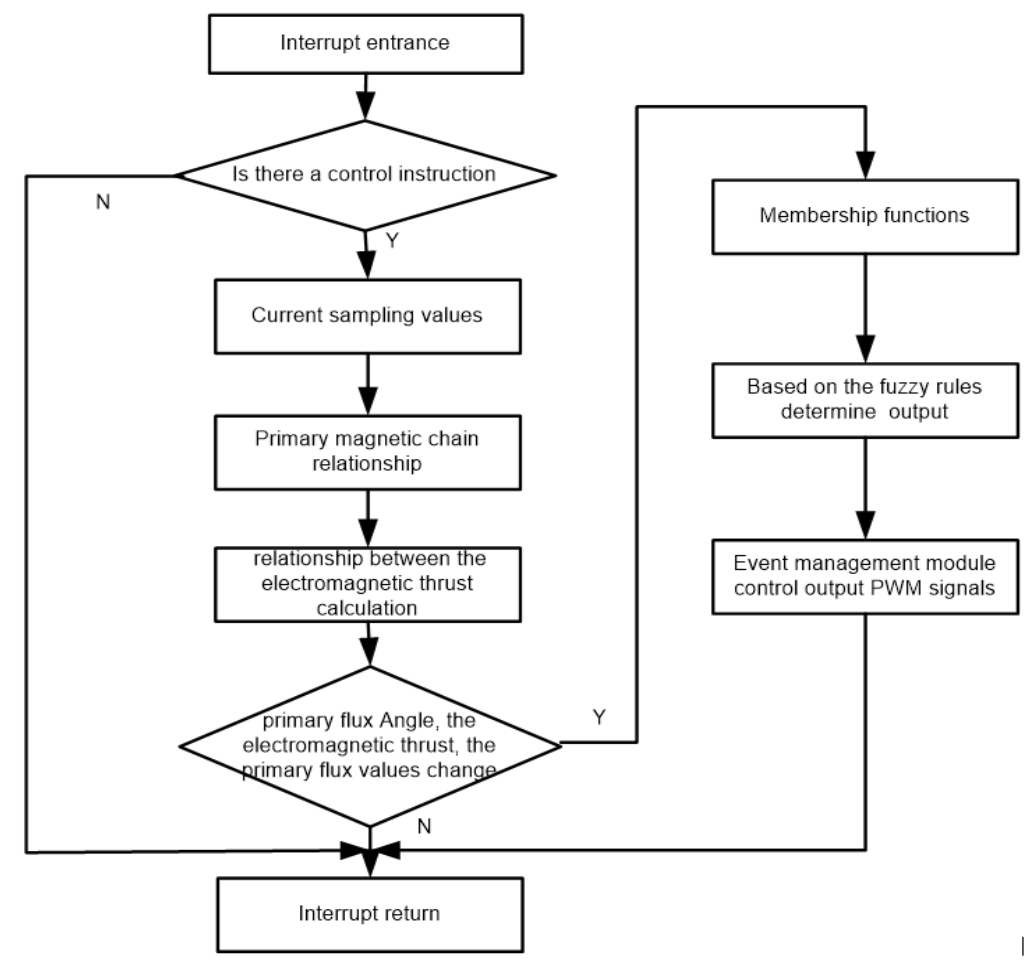

Fig. 6 The software flowchart

However, the problem with inverter control is that every time when the operation state of the upper and lower bridges changes, dead time must be given; or otherwise, the simultaneous conducting from the upper to lower bridges will cause short circuit, or even influence some circuit functions. Therefore, it is critical to design the dead time. Appropriate dead time will guarantee the excellent switching performance of the inverter [10].

\subsection{EXPERIMENT AND SIMULATION OF THE POSITIONING CONTROL SYSTEM}

(1) Sinusoidal input current tracking experiment

The experiment is carried out on a permanent-magnet synchronous linear motor platform LMMZ-0020-075-00 in the laboratory. The technical parameters of the main motor are as follows: $R s=3.9 \Omega$ and $L=1.48 \mathrm{mH}$, the continuous thrust is $60 \mathrm{~N}$, the peak thrust $75 \mathrm{~N}$ and the peak current $4.9 \mathrm{~A}$. Below is the test results of this system. 


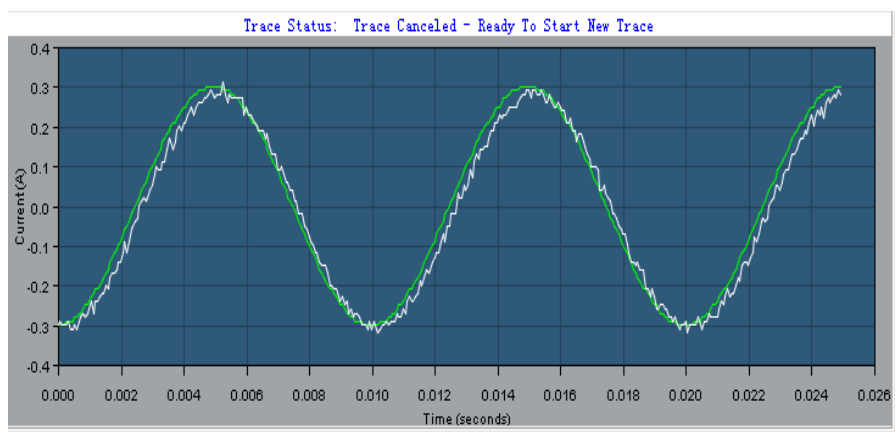

Fig. 7 Sinusoidal input current tracking curve

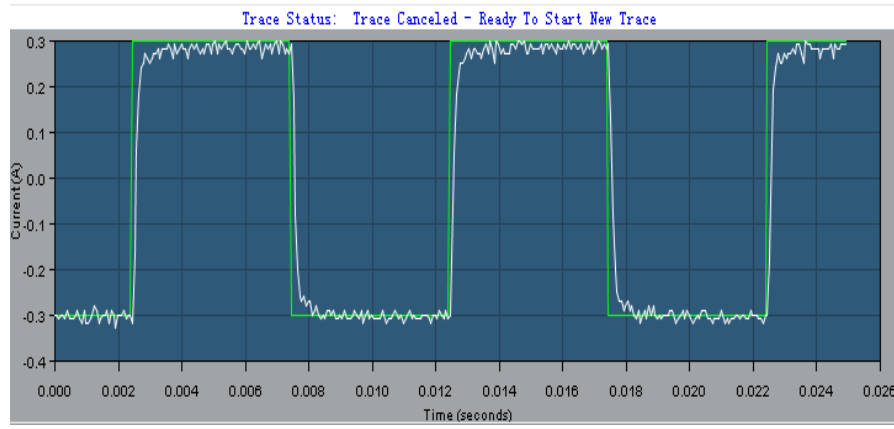

Fig. 8 Square wave input current tracking curve

Figure 7 and 8 show the response curves of the actual system current under the sine wave and square wave inputs, respectively. The input conditions are as follows: the amplitude is $0.3 \mathrm{~A}$, the frequency $100 \mathrm{~Hz}$ and the scope sampling period $67 \mathrm{~ms}$. The white curve in the figure represents the actual output current curve, from which can be seen that the system has a good control effect and that the actual current response curve meets the desired result.

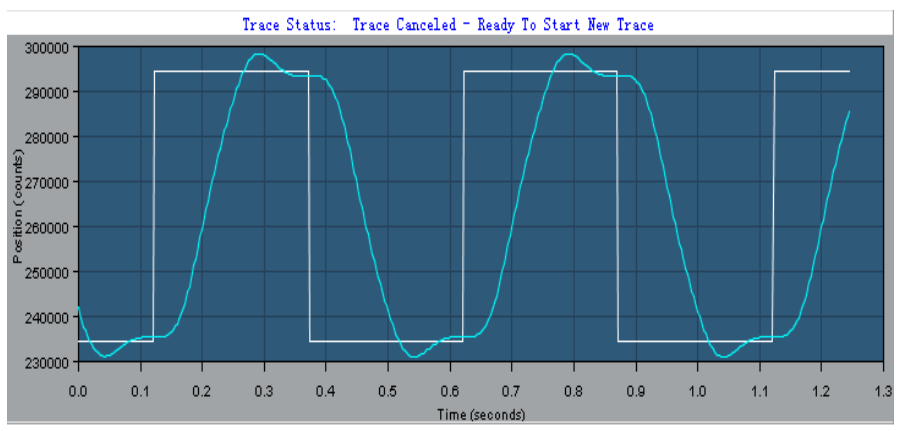

Fig. 9 Experimental curve of the position control system

Figure 9 shows the system position control curve. The input is square waves with a frequency of $2 \mathrm{~Hz}$; the linear motor rotor makes reciprocal motions with a single trip distance of $60 \mathrm{~mm}$; and the scope sampling period is $2 \mathrm{~ms}$. The white curve in the figure is the desired position curve while the blue one shows the actual response curve. According to the figure, the response time of the position control curve is shorter than $0.1 \mathrm{~ms}$, the overshoot is less than $3 \%$, the control delay is relatively small, and the system tracking performance is good. So, it can be seen that the more frequent the linear motor input is, the higher precision the response curve will achieve. 
(2) Simulation of the system model in Simulink

Some tests have been carried out on this system based on the actual motor model. However, considering that it is difficult to measure some parameters, such as thrust, on the testing platform, this paper carries out simulation in Matlab. Below is a simulation model based on this motor platform.

Figure 10 shows an overall simulation model of the permanent-magnet linear synchronous motor servo control system. Combined with a fuzzy controller, this system analyzes the primary magnetic flux deviation, electromagnetic thrust deviation and primary magnetic flux angle as the fuzzy inputs into the fuzzy controller. Then, the system selects the best voltage vector output state based on the fuzzy control rule and directly controls the primary winding of the linear motor to make the output thrust follow the changes of given values. Afterwards, it uses the current and voltage values of the primary winding obtained from the Hall sensor to calculate the next control, thus improving the real-time tracking performance of the whole servo control system.

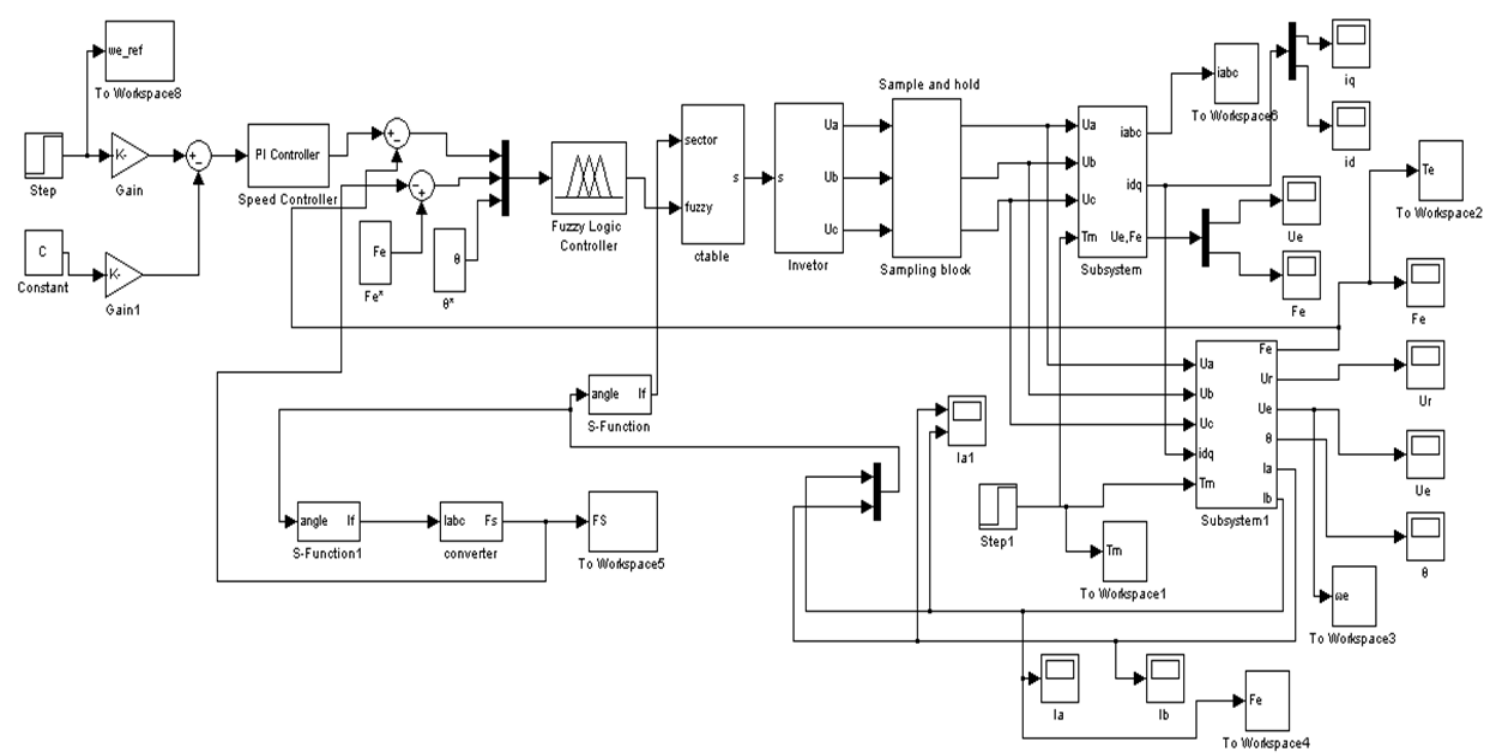

Fig. 10 Overall system control model

When modelling is carried out on the permanent-magnet linear synchronous motor servosystem, it is extremely significant to establish the motor model because the linear motor will serve as the directly-controlled object and reflect the effect of the controller. Any negligence in design will severely affect the controller performance. Therefore, this paper establishes the permanent-magnet linear synchronous motor model in detail, as shown in Figure 11.

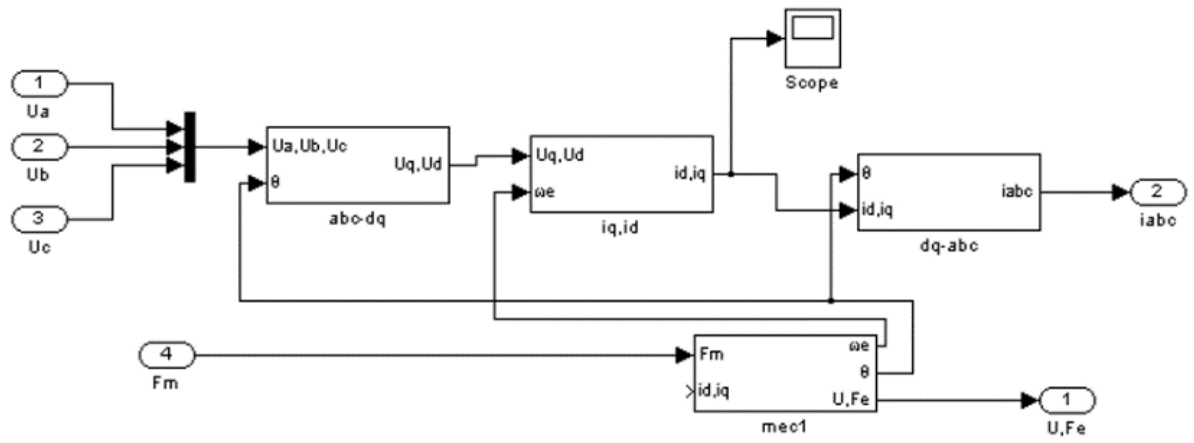

Fig. 11 Permanent magnet linear synchronous motor model 
As shown in Figure 11, the permanent-magnet linear synchronous motor linear model directly reflects the complex relations in the mathematical model of the linear motor, including the conversion of primary parameters from the three-phase coordinate system to the two-phase coordinate system and the reverse conversion as well as the calculation relations between the voltage and current and the primary magnetic flux, electromagnetic thrust and primary magnetic flux angle. This motor system model has laid the foundation for achieving excellent control effect of the controller. Considering that three fuzzy inputs, namely primary magnetic flux deviation, electromagnetic thrust deviation and primary magnetic flux angle, are adopted in the design of the fuzzy controller in this paper, it is very essential to determine these three quantities in the fuzzy control implementation process.

\section{CONCLUSION}

This paper adopts the sliding mode variable structure control algorithm to achieve cooperative positioning control on the dual-axis linear motor on the $X-Y$ platform. In addition, this paper puts forward a high-precision positioning control strategy for linear motor based on the complex dynamic friction model and a contour controller based on friction compensation, which combines the contour error with the tangential error model and the observed state. This design effectively decreases the system contour error and eliminates the impacts of uncertain factors on the system performance through the continuous control of the contour controller, thus making the dual-axis linear motor satisfy the high-precision requirement on the $X$ - $Y$ platform. The simulation results show that the control system conceived in this paper can improve the contour processing precision of the $X$ - $Y$ platform. The more frequent the linear motor input is, the higher precision the response curve will achieve. Compared with other methods, this design has good performance in both speed and precision.

\section{REFERENCES}

[1] Y.J. Liu, T. Li, L.N. Sun, Design of a Control System for a Macro-Microdual-Drive High Acceleration High Precision Positioning Stage for IC Packaging, Science in China, Series E: Technological Sciences, Vol. 52, No. 7, pp. 1858-1865, 2009.

https://dx.doi.org/10.1007/s11431-009-0209-y.

[2] J.H. Kim, J.H. Cho, S.K. Lee, A Design of Hybird Contact Detection Algorithm Forwire Bonder Machine, Proceedings of Industrial Electronics Conference, pp. 2963-2967, https://dx.doi.org/2013.10.1109/IECON.2003.1280719.

[3] P.I. Ro, W. Shim, S. Jeong, Robust Friction Compensation for Sub Micro Meter Positioning and Tracking for a Ball-Screw-Driven Slide System, Precision Engineering, Vol. 24, No. 2, pp. 60-173, https://dx.doi.org/2000.10.1016/S0141-6359(00)00030-1.

[4] Z.Q. Du, Z.D. Zhou, W. Ai, A Linear Drive System for The Dynamic Focus Module of SLS Machine, International Journal of Advanced Manufacturing and Technology, Vol. 32, No. 1112, pp. 1211-1217, https://dx.doi.org/2007.10.1007/s00170-006-0442-5.

[5] L. Bascetta, G. Magnani, P. Rocco, Performance limitations in field-oriented control for asynchronous machines with low resolution position sensing, IEEE Transactions on Control Systems Technology, Vol. 18, No. 3, pp. 559-573, 2010.

https://dx.doi.org/10.1109/TCST.2009.2024300. 
[6] A.M. Bazzi, A. Dominguez-Garcia, P.T. Krein, Markov reliability modeling for induction motor drives under field-oriented control, IEEE Transactions on Power Electronics, Vol. 27, No. 2, pp. 534-546, https://dx.doi.org/ 2012.10.1109/TPEL.2011.2168543.

[7] F. Khoucha, S.M. Lagoun, K. Marouani, Hybrid cascaded H-bridge multilevel-inverter induction-motor-drive direct torque control for automotive applications, IEEE Transactions on Industrial Electronics, Vol. 57, No. 3, pp.892-899, https://dx.doi.org/2010.10.1109/TIE.2009.2037105

[8] Y. Wu, H. Ding, Iterative Learning Variable Structure Controller for High-Speed and HighPrecision Point-to-Point Motion, Robotics and Computer-Integrated Manufacturing, Vol. 24, No. 3, pp. 384-391, 2008. https://dx.doi.org/10.1016/j.rcim.2007.02.021.

[9] A. Johnson Gregg, D. Todd Michael, L. Althouse Bryan, Fiber Bragg grating interrogation and multiplexing with a $3 \times 3$ coupler and a scanning filter, Journal of Light Wave Technology, No. 8, pp. 409-417, 2010. https://dx.doi.org/10.1109/50.857755

[10] M. Mller., H. Hertsch, Temperature on High Voltage Inside Large Power Generators via Use of Fiber Bragg Gratings, Sensors, IEEE, Vol. 1, No. 2, pp. 1603-1607, 2011. https://dx.doi.org/10.1109/ICSENS.2002.1037363.

[11] M.A. Abernethy, P. Brownell, The role of budgets in organizations facing strategic change: an exploratory study. Accounting, Organizations and Society, Vol. 24, No. 3, pp. 189-204, 1999. https://dx.doi.org/10.1016/S0361-3682(98)00059-2. 